\title{
Performance of the ATLAS Liquid Argon Calorimeter with cosmic muons and single LHC beam data
}

Pierre-Simon Mangeard ${ }^{\mathrm{a}}$, on behalf of the ATLAS Liquid Argon Calorimeter Group

${ }^{a}$ Centre de Physique des Particules de Marseille, Aix-Marseille Université, CNRS/IN2P3, Marseille, France

\begin{abstract}
The Liquid Argon (LAr) calorimeter is a key detector in the ATLAS (A Toroidal LHC ApparatuS) experiment at the Large Hadron Collider (LHC), designed to provide precision measurements of electrons, photons, jets and missing transverse energy. Cosmic muon data has been recorded by the LAr calorimeter at various stages of commissioning, with the first single LHC beam data being recorded in September 2008. We present here the LAr calorimeter performance studies based on cosmic muons and LHC beam data. In 2008, 99\% of the LAr readout channels were fully operational. The high energy deposits in the calorimeter readout cells have been used to validate the signal shape reconstruction from calibration predictions at a level better than $\sim 2 \%$. The measured timing alignment agrees with expectations at the $2 \mathrm{~ns}$ level. The non-uniformity of the barrel electromagnetic (EM) calorimeter has been estimated less than $2 \%$ thanks to cosmic muons.
\end{abstract}

Key words:

ATLAS, Liquid Argon sampling calorimeter, performance, cosmic muons

\section{Introduction}

A large effort of in situ commissioning has been undertaken by the ATLAS LAr community (as well as the whole ATLAS collaboration) thanks to cosmic muons and more recently single LHC beam data taken during the first week of LHC operation in September 2008. As a natural follow-up of the commissioning after the beam tests, this represents the final stage to understand the detector before the arrival of the pp collisions data.

After a brief description of the ATLAS LAr calorimeter and its status at the end of 2008, this paper presents data used to study this subdetector. Finally, the main performance is reviewed.

\section{The ATLAS liquid argon calorimeter}

2.1. The subdetector structure

Because it is radiation hard and intrinsically stable and linear, the LAr sampling calorimeter technology has been used for three ATLAS subsystems : i) the Pb/LAr electromagnetic (EM) calorimeter, covering $|\eta|<3.2$, ii) the $\mathrm{Cu} / \mathrm{LAr}$ Hadronic end-cap calorimeters, covering $1.5<$ $|\eta|<3.2$ and iii) the forward $\mathrm{Cu}-\mathrm{W} / \mathrm{LAr}$ forward calorimeter, covering $3.1<|\eta|<4.9$ (See Figure 1).

These systems have been designed to provide precision measurements of electrons, photons, jets and missing

Email address: mangeard@cppm.in2p3.fr (on behalf of the ATLAS Liquid Argon Calorimeter Group) transverse energy thanks to a large angular coverage, an almost perfect hermeticity and a fine granularity. The latter, obtained via a longitudinal and lateral segmentation, leads to a high number of readout channels, 182468 [1], which need to be calibrated and tested before the beginning of pp collisions data taking.

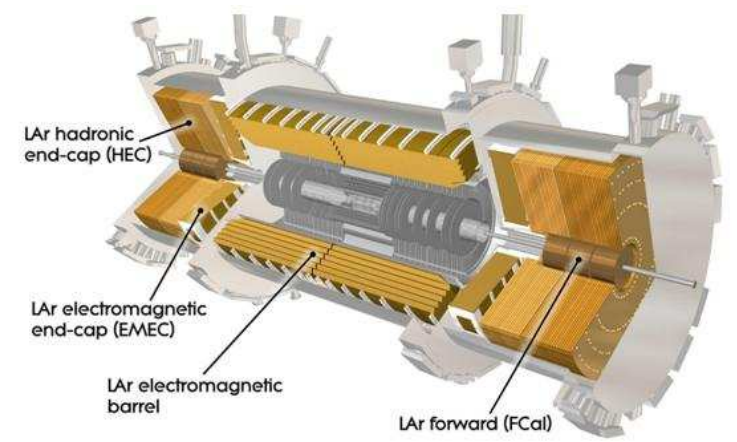

Figure 1: The liquid argon calorimeter systems

\subsection{Status in 2008}

The missing transverse energy is a crucial parameter for many physics studies (top, SUSY), therefore keeping the hermeticity of readout coverage as good as possible is very important. A complete map of dead cells has been extracted from electrical measurements [2] and has been found to affect less than $0.02 \%$ of the cells.

At the end of 2008, only 31 channels were dead and not recoverable which represents $0.017 \%$ of the total number

April 19, 2009 
of channels. This rate is in agreement with the ATLAS requirements. In addition to those permanent dead channels, few readout regions were not functionning because of power supply or front-end electronics problems. That represented less than $0.95 \%$ of the channels, these regions are currently fixed during the shutdown and will be fully operationnal for the pp collisions. Finally, it is worth noting that all the high voltage sectors were powered on and that no zone was dead.

\section{Collected data}

\subsection{Cosmic muons data}

Since Fall 2006, cosmic muons data, first triggered via specially developped trigger boards on Level 1 output of the hadronic tile calorimeter and later with the standard ATLAS Level 1 calorimeter trigger, have been recorded at various stages of the commissioning. Before the arrival of LHC beam, those data were the only one available to check in situ the LAr calorimeter and represent few millions of events.

\subsection{Single $L H C$ beam data}

During the first week of LHC operation in September 2008, with first single beams circulating in the LHC, events resulting from dump of the beam on collimator, called later 'splash events', occuring $140 \mathrm{~m}$ from the ATLAS point interaction, were recorded. As a consequence, a huge particule flow, mainly muons and pions, went trough the detector and several hundreds of $\mathrm{TeV}$ were deposited over the whole coverage of each LAr calorimeter samplings. Figure 2 presents the accumulated energy per cell in the main sampling of the EM calorimeter over 100 beam splash events. This dataset is the first opportunity to check the signal reconstruction and timing alignment over the total spatial coverage of the LAr calorimeter.

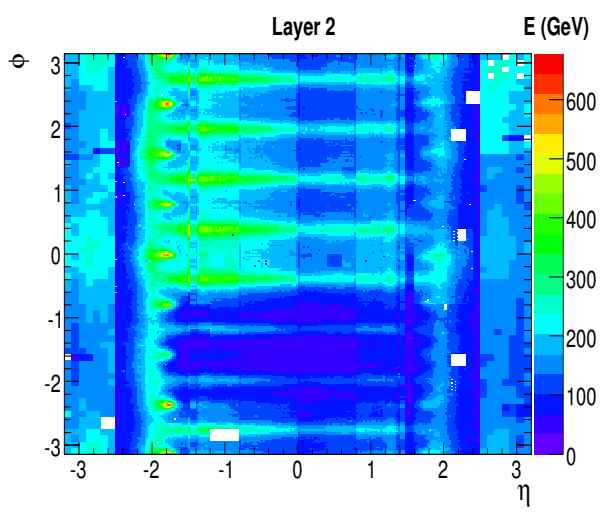

Figure 2: $(\eta, \phi)$ map of deposited energy in the main EM layer, accumulated over 100 beam-collimator splash events. The white regions correspond to cells masked because of readout or calibration issues.

\section{Performance of the LAr Calorimeter}

\subsection{Signal reconstruction}

The high energy deposits in the readout cells, induced either by catastrophic energy loss of the cosmic muons or by beam interactions are used to validate the signal shape of EM calorimeter response derived from the calibration pulse. Figure 3 shows the agreement between a typical ionization pulse shape (triangle) and its prediction (dots) which is better than $2 \%$ over the full pulse duration.

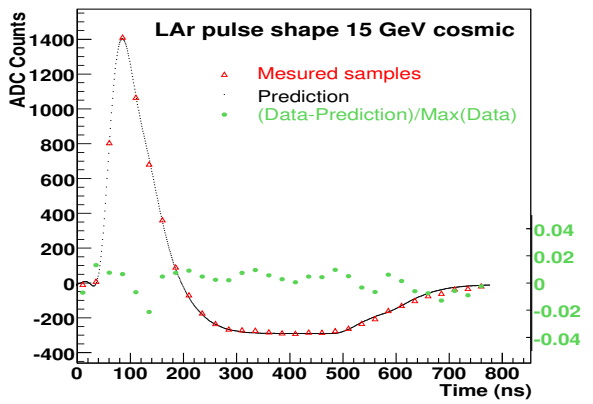

Figure 3: Ionization pulse shape of a $15 \mathrm{GeV}$ cosmic signal reconstructed in the EM barrel calorimeter (triangle) compared the prediction (dots). Their relative difference with respect to the maximal amplitude is represented by the full circles.

The study of the 32-sample measured pulses of cosmic events allows to assess the drift time in the liquid argon for every cell. The undershoot of the pulse is related to the drift time and the rising at the end of the pulse is sensitive to a shift of the electrode with respect to its nominal central positioning. Figure 4 presents the drift time (black dots) in the middle sampling of the EM barrel calorimeter as a function of the pseudo-rapidity. Data agree well with the expected values (grey line) derived from the structure of the absorbers. The contribution of the gap variation to the barrel calorimeter response non-uniformity is no larger than $0.3 \%$.

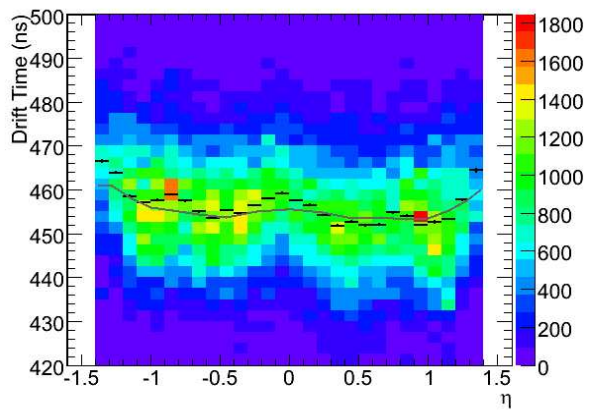

Figure 4: Drift time in liquid argon, determined with high energy cosmic signals (dots), in function of the pseudo-rapidity for the EM barrel calorimeter. The line corresponds to the expected values. 
In spite of the non nominal value of the high voltage $(<25 \%)$ in the forward calorimeter, ionization pulse shapes in each of its samplings have been observed in the single LHC beam data.

\subsection{Timing}

The coherent arrival of particle flow through the whole detector, induced by beam splash events, allows us to study the timing of the whole LAr calorimeter. Figure 5 shows the relative timing alignment, deduced from data (dots), compared to prediction (squares), extrapolated from calibration timing and readout path, for one side of the EM barrel. A agreement at a level of $\sim 2 \mathrm{~ns}$ is observed.

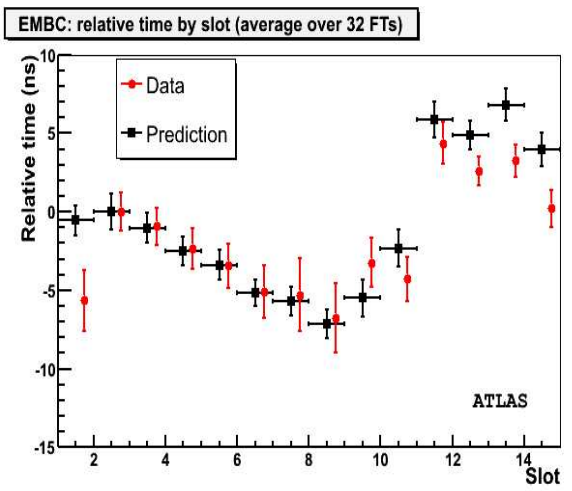

Figure 5: Comparison of the timing alignment of one side of the EM barrel from beam-collimator splash events (dots) with predictions (squares). A "Slot" is a group of readout cells in eta region of a sampling. Distribution is averaged over the azimutal angle $\phi$.

\subsection{Energy response}

The energy resolution of the LAr calorimeter can be written as : $\frac{\sigma(E)}{E}=\frac{a}{\sqrt{E(\mathrm{GeV})}} \oplus \frac{b}{E(\mathrm{GeV})} \oplus c$ where $a$ is the sampling term, $b$ is the noise term and $c$ the constant term, dominant at high energy. For the EM calorimeter, very strong constraints on $\mathrm{c}(<0.7 \%)$ are required in order to optimize the $\mathrm{H} \rightarrow \gamma \gamma$ and new heavy gauge boson searches.

The first cosmic muons data taken in 2006 and in March 2007 allowed to evaluate in situ the energy response of the EM barrel [3]. Despite the low signal $(\sim 300 \mathrm{MeV}$ per muon), the energy agrees statistically with the MonteCarlo (MC) in an overall energy scale around $3 \%$. As cosmic muons are minimum ionizing particles, the nonuniformity of the energy response, which contributes significantly to the constant term, has been compared to the cell depth of the layer of the EM calorimeter where the energy is mainly deposited. The response non-uniformity was shown to be less than $2 \%$ in $0.1 \eta$ bins in the region $-0.8<\eta<0.8$ as presented in Figure 6 . The result obtained with cosmic muons is limited by statistic and is coherent with published results from test beam [4]. A similar study is currently performed with a much higher statistics $\left(\sim 2 \times 10^{6}\right.$ events $)$.

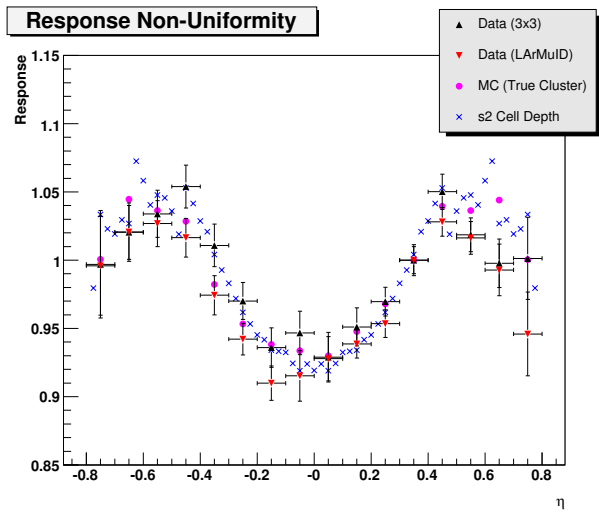

Figure 6: Normalized $\eta$ dependance of the energy response to cosmic muons of the EM barrel calorimeter. Two cluster algorithms, of Monte-Carlo and cell depth evolution of the main EM layer.

\section{Conclusion}

Since 2006, the liquid argon calorimeter of ATLAS has been using cosmic muons and single LHC beam data to assess the performance of the subdetector. This is necessary in order to optimize the use of the first LHC pp collisions data. At the end of 2008 , about $99 \%$ of the readout channels were fully operationnal and the remaining $1 \%$ is fixed during the present shutdown. No dead region should affect the missing transverse energy measurement. The particle flow occured during the beam-collimator splash allowed to verify the timing aligment at the level of 2 ns. Finally, the non-uniformity of the EM calorimeter barrel has been checked at the level of $2 \%$.

The results are promising and show that the LAr calorimeter is in a very good shape before the first pp collisions.

\section{Acknowledgments}

The work presented in this paper is the result of the collaboration of many people within the ATLAS liquid argon calorimeter group over many years. I especially would like to thank people who operate daily the detector, without them, the results shown here would not be possible.

\section{References}

[1] The ATLAS Collaboration, G. Aad et al., The ATLAS Experiment at the CERN Large Hadron Collider, JINST 3 (2008) S08003.

[2] ATLAS Electromagnetic Liquid Argon Calorimeter Group, Construction, assembly and tests of the ATLAS electromagnetic barrel calorimeter, Nucl. Inst. Meth. A 558 (2006) 388.

[3] M. Cooke, P.S. Mangeard, M. Plamondon et al., In situ commissioning of the ATLAS electromagnetic calorimeter with cosmic muons, ATLAS NOTE ATL-LARG-PUB-2007-13.

[4] M. Aharrouche et al., Response Uniformity of the ATLAS Liquid Argon Electromagnetic Calorimeter, Nucl. Inst. Meth. A 582 (2007), 429-455. 